\title{
A RARE CASE OF SPACE OCCUPYING LESION OF BRAINSTEM IN AN ELDERLY MALE PATIENT

\author{
Jayanth S S ${ }^{1}$, Ambali A $\mathrm{P}^{2}$, M S Mulimani ${ }^{3}$
} \\ ${ }^{1}$ Junior Resident, ${ }^{2}$ Professor, Department of General Medicine, BLDEU's Shri B M Patil Medical College and Hospital, Vijayapur, Karnataka, India.
}

\section{ABSTRACT}

An elderly male patient aged 65 presented to us with history of swaying towards left side of the body since 1 month with normal higher mental functions and neurological examination suggestive of cerebellar ataxia. MRI Brain plus contrast was suggestive of an irregular, ill-defined heterogeneous enhancing lesion with few necrotic areas within and few foci of blooming on FFE (Fast Field Echo imaging technique) with significant perilesional oedema involving right thalamus and brainstem showing Choline peak on MR Spectroscopy.

KEYWORDS: Neurological examination; Cerebellar ataxia; MRI brain; FFE.

\section{INTRODUCTION}

Brainstem tumours are defined as lesions which occur in the midbrain, the pons, or the medulla oblongata. The tumour's extension is considered focal when it occupies $<50 \%$ of the axial brainstem diameter, and the extension is considered diffuse when the lesion is poorly demarcated and is $>50 \%$ of the brainstem diameter [1-3]. Brainstem glioma is the most frequent tumour of the region. A clear bimodal age distribution supports the distinction between brainstem gliomas in children and adults. In contrast with the paediatric population in which brainstem gliomas represent up to $20 \%$ of brain tumours and exhibit a rather homogeneous and unfavourable course, adult brainstem gliomas are rare (accounting for only $1 \%-2 \%$ of adult brain gliomas) and heterogeneous with varying radiological patterns and variable prognosis[4,5]. Histologically, adult brainstem gliomas can have an astrocytic, oligodendroglial, or mixed appearance, with astrocytic tumours further characterized as either pilocytic or diffusely infiltrative.

\section{CASE REPORT}

An elderly male patient aged 65 presented to casualty with complaints of swaying towards the left side of the body since 1 month. The swaying was minimal during the initial days of illness; the patient could walk with the support of fellow being and has progressed over one month to an extent where patient was not able to walk even with support. There was no history of vertigo

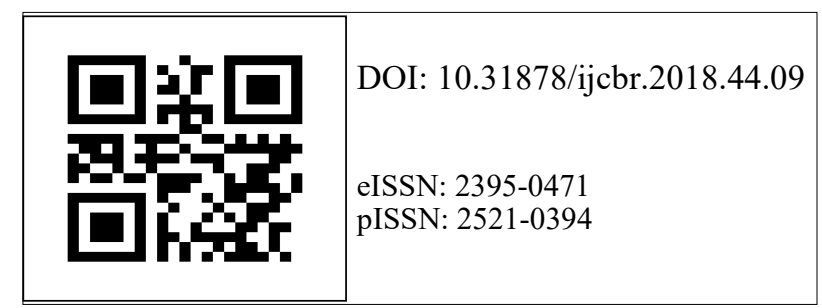

or speech abnormalities. The patient was able to sit with support. Patient was a tobacco chewer and smoker. He was not a known case of Hypertension, Type 2 Diabetes Mellitus, and Ischemic Heart Disease. On examination patient was conscious, oriented and vitals were stable. Neurological examination showed normal higher mental functions. Cranial nerves and motor system were intact. Sensory system was intact. Cerebellar system revealed up-beating nystagmus at primary gaze; impaired finger nose test, heel-shine test, dysdiadokinesia towards the left.

Neuroimaging was done for the patient along with other relevant blood investigations. MRI Brain plain plus contrast was suggestive of: 1) An irregular, ill-defined lesion appearing isointense on T2/ FLAIR \& hyper intense on T1 showing restricted diffusion on DWI and few foci of Blooming on FFE with significant perilesional oedema is noted involving right thalamus, midbrain, pons (predominantly on right side) and right cerebellar peduncle. 2) On contrast administration, the lesion is showing heterogeneous enhancement with few non enhancing necrotic areas within. 3) MR Spectroscopy (MRS) shows Choline peak.4) Another small well defined enhancing lesion with surrounding oedema is noted in left temporal lobe. 5) Chronic lacunar infarct is noted in left lentiform nucleus.6) Mild prominent cerebral sulci, basal cisterns, sylvian fissures, ventricles and cerebellar folliae are noted. 6) Multiple white matter hyperintensities (FLAIR) are noted in bilateral periventricular region and bilateral centrum semiovale - suggestive of chronic ischemic changes.

Fundoscopy showed no evidence of papilledema. USG Abdomen showed no sonologically significant abnormality. Chest X-ray posterior-anterior view showed features of emphysematous chest.

Corresponding author: Dr. Jayanth S S, Junior Resident, Department of General Medicine, BLDEU's Shri BM Patil Medical College and Hospital, Solapur road, Vijayapur-586103. Email: Jayanthss92@gmail.com 
Table 1. Shows various investigations done and their results

\begin{tabular}{|c|c|}
\hline INVESTIGATIONS & RESULT \\
\hline HIV Rapid & Negative \\
\hline Blood Glucose & $159 \mathrm{mg} / \mathrm{Dl}$ \\
\hline Serum Creatinine & $1.0 \mathrm{mg} / \mathrm{dL}$ \\
\hline Serum Sodium & $135 \mathrm{mmol} / \mathrm{L}$ \\
\hline Serum Potassium & $3.8 \mathrm{mmol} / \mathrm{L}$ \\
\hline Total Leucocyte Count & $6570 \mathrm{cells} / \mathrm{cmm}$ \\
\hline Hemoglobin & $14.0 \mathrm{gm} \%$ \\
\hline RBC & $4.74 \mathrm{millions} / \mathrm{cmm}$ \\
\hline ESR & 20 \\
\hline Platelet & 2.69 lakhs/cmm \\
\hline
\end{tabular}

\section{DISCUSSION}

On evaluating for the space occupying lesion of the brain by neuroimaging and other relevant investigations to rule out other systemic involvement and infective pathology, differential diagnosis considered were 1) High grade glioma 2) Multicentric glioma 3 ) Glioma with metastasis. Glioma with metastasis was ruled out as the history, clinical examination and investigations revealed no evidence of tumour elsewhere in the body. High grade gliomas are malignant, often rapidly progressive brain tumours that are divided into anaplastic gliomas and glioblastoma based on their histologic and molecular features. Multicentric gliomas are widely separated lesions whose simultaneous presence cannot be attributed to dissemination through commissural pathways, CSF channels, and blood or by local extension through satellite formation [6]. As neuroimaging in this case does not show any evidence of disseminated lesions, high grade glioma can be considered as our probable diagnosis. Conventional radiotherapy is the standard treatment for diffuse intrinsic low-grade brainstem gliomas in adults (the median survival is 5 years)[6].

\section{CONCLUSION}

Differential diagnosis considered were High grade glioma, multicentric glioma and Glioma with Metastasis and we speculate that this patient had high grade glioma. Despite significant advances in neuroradiology techniques, a purely radiological classification remains imperfect in the absence of a histological diagnosis. A biopsy may often be reasonably avoided in the diffuse non-enhancing forms, obtaining histological proof seems necessary in many contrast-enhanced brainstem lesions because of the wide variety of differential diagnosis in adults.

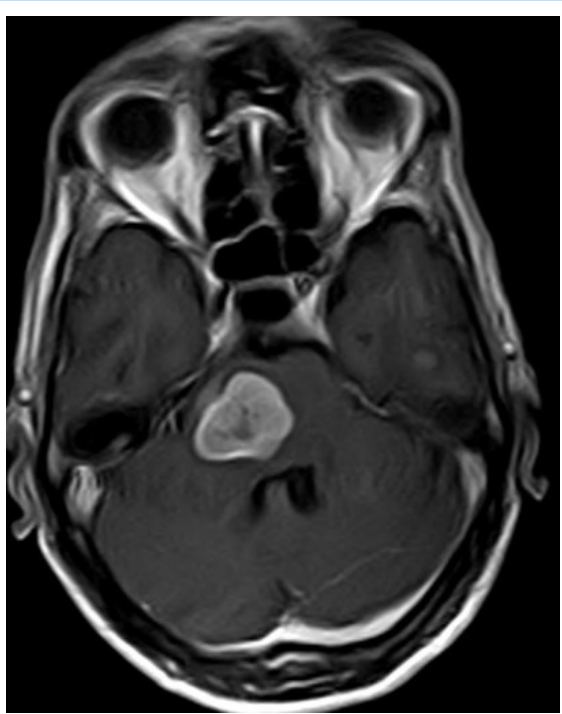

Fig 1. Irregular, ill-defined lesion appearing isotense on T2/ FLAIR

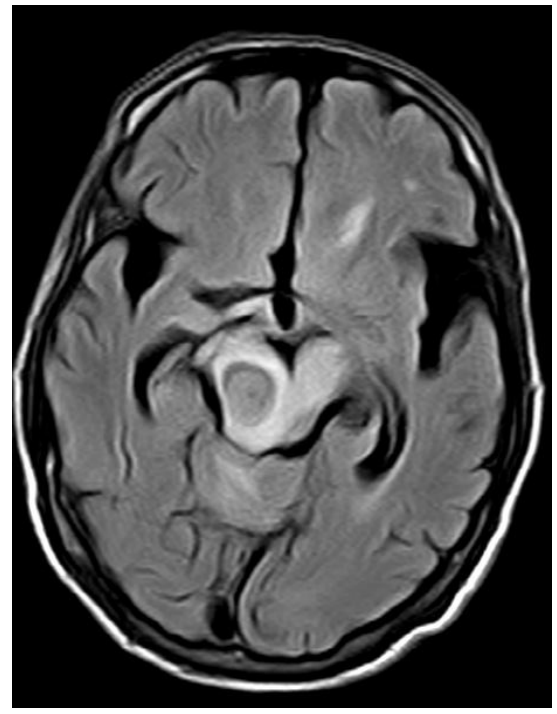

Fig 2. Heterogenous enhancement with few non enhancing necrotic areas within

\section{REFERENCES}

1) Barkovich A, Krischer J, Kun L, Packer R, Zimmerman R, Freeman C et al. Brain Stem Gliomas: A Classification System Based on Magnetic Resonance Imaging. Pediatric Neurosurgery. 1990;16(2):73-83.

2) Donaldson S, Laningham F, Fisher P. Advances Toward an Understanding of Brainstem Gliomas. Journal of Clinical Oncology. 2006;24(8):1266-1272.

3) Fischbein N, Prados M, Wara W, Russo C, Edwards M, Barkovich J. Radiologic Classification of Brain Stem Tumors: Correlation of Magnetic Resonance Imaging Appearance with Clinical Outcome. Pediatric Neurosurgery. 1996;24(1):9-23.

4) Guillamo J. Brainstem gliomas in adults: prognostic factors and classification. Brain. 2001;124(12):25282539. 
5) Landolfi J, Thaler H, DeAngelis L. Adult brainstem gliomas. Neurology. 1998;51(4):1136-1139.

6) Reyes-Botero G, Mokhtari K, Martin-Duverneuil N, Delattre J, Laigle-Donadey F. Adult Brainstem Gliomas. The Oncologist. 2012;17(3):388-397.

How to Cite this article: Jayanth S S, Ambali A P, M S Mulimani. A rare case of space occupying lesion of Brainstem in an Elderly Male Patient. Int. j. clin. biomed. res. 2018;4(4): 39-41. 